\title{
Severe Coronavirus Disease 2019 during pregnancy: a case report study
}

\author{
Ying $\mathrm{Yu}^{1}$, Cuifang Fan ${ }^{1}$, Junmei Bian ${ }^{2}$, Yin Shen ${ }^{1}$, and Xingxing Bao ${ }^{2}$ \\ ${ }^{1}$ Renmin Hospital of Wuhan University \\ ${ }^{2}$ Tongren Hospital of Wuhan University
}

April 28, 2020

\section{Case}

A 35-year-old pregnant woman, 34 weeks gestation, was admitted to a tertiary hospital after 3 hours of clear vaginal fluid discharge. Labor occurred spontaneously on that day, and a healthy female baby was delivered with no evidence of infection (SARS-CoV-2 negative upon throat swab). This was the mother's second pregnancy and delivery. On the second day after vaginal delivery, the patient presented with persistent low fever and dry cough. Laboratory investigations showed white blood cell count (WBC) $7.1 \times 10^{9} / \mathrm{L}$ (reference range 3.5-9.5), neutrophil ratio (N\%) 84.7\% (reference range 40-75), lymphocyte count (L\#) 0.69x10 $10^{9} \mathrm{~L}$ (reference range 1.1-3.2), and C-reactive protein (CRP) $73.63 \mathrm{mg} / \mathrm{L}$ (reference range 0.0-0.4). Throat swabs from the patient tested positive for SARS-CoV-2 by real-time RT-PCR assays, and the chest CT scan showed multiple infiltrations of different sizes in both lungs and a small amount of fluid on both sides of the chest. Considering these findings, the patient was diagnosed with SARS-CoV-2 and isolated. She had no other history of comorbidities, and was treated with i.v. antibiotics and hormones, and was administered oxygen through a nasal catheter (Figure 1). The patient experienced dyspnea and cyanosis on the following day. Considering her critical condition, she was transferred to the intensive care unit (ICU) for further treatment.

After transferring to the ICU, she quickly developed severe acute respiratory distress syndrome (ARDS), and her symptoms did not improve after she was given a non-invasive ventilator. The CT scan showed a density shadow and a large-scale ground-glass opacity in both lungs, which had progressed in severity compared with the scan on Day 2 post-delivery. She required tracheal intubation on the 14th day of hospitalization. Gram-positive and gram-negative bacteria (Acinetobacter baumannii, Klebsiella pneumoniae) were positively cultured in her sputum. Antibiotics were administered according to the results of a drug sensitivity test. Empirical antifungal treatment was also given. Antiviral, nutritional and symptomatic treatments were also performed simultaneously. After 11 days of ICU treatment, the patient's condition significantly improved. Tracheal intubation was removed and replaced with non-invasive ventilator. The patient was changed to high-flow humidified oxygen several days later, and she was transferred to the general ward for treatment. Her symptoms and primary treatment strategy are illustrated as shown in Figure 1.

\section{Discussion}

Physiological adaptive changes, increased oxygen consumption, and suppressed immune state increase the susceptibility of pregnant women to respiratory pathogens and pneumonia ${ }^{1}$. Previous reports have shown that HPV, HIV, and other viruses are related to increased rate of preterm birth ${ }^{2,3}$. However, whether preterm delivery described in this report was caused by SARS-CoV-2 remains unknown. The case in the present report is a pregnant woman infected with COVID-19 that rapidly developed into severe respiratory infection following vaginal delivery. Since there is only one case in the present report, it is difficult to 
draw conclusions with regard to the etiology of the symptoms after vaginal delivery. Whether the strong contractions during natural delivery can exacerbate pneumonia associated with COVID-19 still needs to be confirmed. Huijun et. al. have reported that none of nine confirmed patients infected with SARSCoV-2 developed severe COVID-19 pneumonia or died after the caesarean section in their third trimester ${ }^{4}$, suggesting that cesarean section may carry less risk in pregnant women infected with SARS-CoV-2. Due to the increased oxygen consumption and pulmonary load during pregnancy, cesarean section may help to avoid adverse clinical consequences caused by pulmonary insufficiency and also reduces the possibility of maternal viremia during delivery. In addition, we didn't observe any abnormalities from the newborn baby, which indicated no mother-to-child transmission by vaginal delivery. This observation was consistent with Huijun and colleagues' finding of no intrauterine transmission of COVID-19.

\section{Funding}

No funding.

\section{Acknowledgement}

The authors thank medical staff of department of Obstetrics and Gynecology of Renmin Hospital of Wuhan University, department of Pediatrics of Tongue Hospital of Wuhan University.

\section{Disclosure of Interest}

The authors have no conflict of interest to declare. Completed disclosure of interest forms are available to view online as supporting information.

\section{Contribution to authorship}

CF participated in the design and plan of the study. JB and XB contributed to the data collection. YY drafted the manuscript. CF and YS revised the manuscript critically. All authors read and approved the final manuscript.

\section{Details of ethical approval}

This study was approved by the Clinical Research Ethics Committee of Renmin Hospital Wuhan University (Grant No. WDRY2020-K016), and written consent to publish was obtained from the patient.

\section{References}

1. Kohlhepp LM, Hollerich G, Vo L, et al. [Physiological changes during pregnancy]. Anaesthesist. 2018;67(5):383-396.

2. Feist H, Hussein K, Stucki-Koch A, et al. Villitis of unknown etiology and chronic deciduitis are not associated with human papilloma virus and enterovirus infection. Virchows Arch. 2020.

3. Gupta A, Montepiedra G, Aaron L, et al. Isoniazid Preventive Therapy in HIV-Infected Pregnant and Postpartum Women. N Engl J Med.2019;381(14):1333-1346.

4. Jain SH, Baackes J, O'Connell JJ. Homeless Special Needs Plans for People Experiencing Homelessness. JAMA. 2020.

\section{Hosted file}

Figure legend.docx available at https://authorea.com/users/314222/articles/444564-severe-coronavirusdisease-2019-during-pregnancy-a-case-report-study 